\title{
Cloning and Expression of the Defective Genes from a Patient with $\delta$-Aminolevulinate Dehydratase Porphyria
}

\author{
Nobuhiro Ishida, * Hiroyoshi Fujita," Yoshiaki Fukuda," Teruhisa Noguchi," Manfred Doss," \\ Attallah Kappas, * and Shigeru Sassa* \\ *The Rockefeller University, New York $10021 ;{ }^{\ddagger}$ The Suntory Institute for Biomedical Research, Osaka, Japan; \\ and ${ }^{\S}$ Philipps University, Marburg, Federal Republic of Germany
}

\begin{abstract}
Cloning and expression of the defective genes for $\delta$-aminolevulinate dehydratase (ALAD) from a patient with inherited ALAD deficiency porphyria (ADP) were carried out. Cloning of CDNAs for the defective ALAD were performed from EBVtransformed lymphoblastoid cells of the proband, and nucleotide sequences were determined. Two separate point mutations resulting in a single amino acid change in each ALAD allele were identified. One, $C^{718} \rightarrow T$, termed ' $G 1$ ', occurred in the allele within the substrate-binding site, producing an $\mathbf{A r g}^{240} \rightarrow$ Trp substitution; the other, $\mathbf{G}^{820} \rightarrow$ A, termed ' $G 2$ ', occurred downstream of this site in the other allele, resulting in an $\mathrm{Ala}^{274}$ $\rightarrow$ Thr substitution. Using the reverse transcription-polymerase chain reaction, the mother, the brother, and the sister were shown to have the G1 defect. Expression of the G1 cDNA in Chinese hamster ovary cells produced ALAD protein with little activity; the G2 cDNA produced the enzyme with $\approx 50 \%$ normal activity. Pulse-labeling studies demonstrated that the G1 enzyme had a normal half life, while the G2 enzyme had a markedly decreased half life. These data thus define the separate point mutations in each ALAD allele, as well as the altered properties of the two enzymic proteins encoded by the mutant genes in a patient with ADP. (J. Clin. Invest. 1992. 89:14311437.) Key words: Point mutation • PCR • transfection • expression • compound heterozygosity
\end{abstract}

\section{Introduction}

$\delta$-Aminolevulinate dehydratase (E.C.4.2.1.24; ALAD) ${ }^{1}$ is a cytosolic enzyme in the heme biosynthetic pathway that catalyzes the condensation of two molecules of $\delta$-aminolevulinic acid to form a monopyrrole, porphobilinogen (1). The enzyme activity is present in great excess in normal cells, thus a partial deficiency of this enzyme activity is not accompanied by any clinical consequences (2-4). However, a marked enzyme deficiency may occur through inhibition of the enzyme activity by certain

Address correspondence and reprint requests to Dr. Shigeru Sassa, The Rockefeller University, New York, NY 10021.

Received for publication 27 August 1991 and in revised form 3 December 1991.

1. Abbreviations used in this paper: ADP, ALAD deficiency porphyria; ALAD, delta-aminolevulinate dehydratase; A/M, activity/mass; ASOs, allele-specific oligonucleotides; $\mathrm{CHO}$, Chinese hamster ovary; CRIM, cross-reactive immunological material; $\mathrm{PCR}$, polymerase chain reaction; RT-PCR, reverse transcription PCR.

J. Clin. Invest.

(C) The American Society for Clinical Investigation, Inc.

0021-9738/92/05/1431/07 \$2.00

Volume 89, May 1992, 1431-1437 chemicals, e.g., lead (5), succinylacetone $(6,7)$, as well as by an inherited ALAD deficiency $(8,9)$, which may result in the development of a clinically severe hepatic porphyria $(3,4)$. Four unrelated cases of inherited ALAD porphyria (ADP) have been reported to date (9). In our previous study (10), we described a molecular defect in an ALAD allele, termed ' $G 2$ ', in an adultonset proband with ADP, originally reported from Germany (8). This finding represented the first demonstration of a mutation in the human ALAD gene, and suggested that the proband had two separate point mutations in the ALAD gene (10). In the present study, we describe the molecular analysis of the enzymatic defect encoded by the other ALAD allele in the proband, termed ' $\mathrm{Gl}$ ', and ALAD phenotype studies in the proband's family using allele-specific oligonucleotides (ASOs) for the $\mathrm{G} 1$ and the $\mathrm{G} 2$ mutations. In addition, we report results on the activity and the turnover rates of the mutant enzymes expressed by the $\mathrm{G} 1$ and the $\mathrm{G} 2 \mathrm{cDNAs}$ in Chinese hamster ovary (CHO) cells. This study thus represents the first complete molecular analysis of the aberrant enzymes expressed by both mutant ALAD alleles in a patient with ADP.

\section{Methods}

Patient and family members studied. The patient developed signs and symptoms of ADP at the age of 15 (8). His mother, a brother, and a sister studied were all from family B, described previously (8).

Cell cultures. Isolation of lymphocytes, transformation of cells with EBV, and cultivation of lymphoblastoid cells were carried out, as described previously (9).

Synthesis of $A L A D C D N A$. Poly(A) ${ }^{+}$RNA was prepared from EBVtransformed lymphoblastoid cells, as described previously $(11,12)$. The first strand of CDNA was synthesized using Moloney Murine Leukemia Virus (MMLV) reverse transcriptase (Pharmacia Fine Chemicals) (SuperScript $\mathrm{RNaseH}^{-}$reverse transcriptase; Gibco-BRL, Gaithersburg, MD) primed with oligo(dT) $)_{12-18}$ (Pharmacia Fine Chemicals, Uppsala, Sweden).

Polymerase chain reaction (PCR). Two oligomers for amplification, i.e., ALAD1: 5'-CCGGAATTCCAACCAACTGATGCCC, and ALAD2: 5'-GTTCTAGAGCCTGGCACTGTCCTCC, were designed with the EcoRI site and the Xbal site at the 5'-terminus, respectively (10). These oligomers correspond to the 5'-untranslated and the 3 '-untranslated regions of ALAD cDNA (13), respectively. Amplifications were performed three times in separate experiments, and further steps were processed separately as well. PCR was carried out using a DNA Thermal Cycler (Perkin-Elmer-Cetus Corp. Norwalk, CT), employing a thermal cycle program, as described previously (10).

Cloning and sequencing of $c D N A s$. PCR products were digested with EcoRI and Xbal, and cloned into pGEM4z vector (Promega Biotec, Madison, WI). Appropriate restriction fragments were subcloned into $\mathrm{M} 13 \mathrm{mp} 18$ or M13mp19. DNA sequencing was carried out by the dideoxy chain-termination method using a genetically engineered $\mathrm{T} 7$ DNA polymerase (Sequenase version 2.0; United States Biochemical Corp., Cleveland, $\mathrm{OH})$ (14-16). Sequence data were analyzed using DNASIS software (Hitachi, San Bruno, CA).

Phenotype analysis. ALAD phenotype analyses in the proband's 
family were carried out using the reverse transcription-polymerase chain reaction (RT-PCR) (10). Purification of total RNA, synthesis of cDNA, amplification of ALAD cDNA, and hybridization with ASOs were performed, as described previously (10). Briefly, total RNA (1-5 $\mu \mathrm{g})$ was reverse transcribed with SuperScript $\mathrm{RNaseH}^{-}$reverse transcriptase, using random hexamers (Pharmacia Fine Chemicals) as a primer. ALAD cDNAs were then amplified by PCR, using ALAD1 and ALAD2 as the primers. After 45 cycles of amplification, the aliquots of RT-PCR products were subjected to hybridization with ASOs, or to direct sequencing.

Direct sequencing of $R T-P C R$ products of $A L A D$ mRNA. Singlestranded DNAs were synthesized from aliquots of RT-PCR products, using asymmetric PCR according to Gyllensten et al. (17). The reaction mixture, which included ALAD1 and ALAD2 as the specific primers at the molar ratio of either $100 \mathrm{pmol} / 5 \mathrm{pmol}$ or $5 \mathrm{pmol} / 100 \mathrm{pmol}$, was subjected to the following amplification cycles, using a Biocycler Oven (BIOS Corp., New Haven, CT): 3 cycles of $1 \mathrm{~min}$ at $94^{\circ} \mathrm{C}, 30 \mathrm{~s}$ at $57^{\circ} \mathrm{C}$, and $45 \mathrm{~s}$ at $72^{\circ} \mathrm{C}$, followed by 27 cycles of $30 \mathrm{~s}$ at $94^{\circ} \mathrm{C}, 30 \mathrm{~s}$ at $57^{\circ} \mathrm{C}$, and $45 \mathrm{~s}$ at $72^{\circ} \mathrm{C}$. Amplified products were purified using Qiagen column (Qiagen Inc., Chatsworth, CA). Aliquots of the purified products were used as a template for direct sequencing. DNA sequencing was carried out by the dideoxy chain-termination method, using appropriate oligomers as a sequencing primer (14-16).

Expression of $A L A D$ cDNAs. pdKCR-Neo, the vector used for the expression of ALAD cDNAs, is a derivative of the expression vector pKCR (18), which contained the SV40 early gene promoter, donor, and acceptor splice sites derived from the rabbit $\beta$-globin gene, as well as polyadenylation sites derived from the rabbit $\beta$-globin gene, and the SV40 early gene. The construction of pdKCR-Neo was performed by a serial modification (19-21) of the plasmid, and by replacing the $\mathrm{dhfr}$ gene with the neomycin resistant gene (S. Oikawa, personal communication). The resulting plasmid contained a unique EcoRI site for insertion of foreign sequence (19), into which the EcoRI fragments of cDNAs were inserted. Recombinant plasmids, which encode the normal ALAD, a mutant ALAD with the Gl defect, and a mutant ALAD with the $\mathrm{G} 2$ defect, were introduced into $\mathrm{CHO}$ cells by coprecipitation with calcium phosphate (CellPhect Transfection kit; Pharmacia Fine Chemicals). Selection with G418 (Geneticin; Gibco-BRL) $(1,600 \mu \mathrm{g} /$ $\mathrm{ml}$ ) was carried out, starting $48 \mathrm{~h}$ after transfection.

Western blot analysis. Human ALAD expressed in CHO cells was specifically detected by Western blot analysis, using $0.5-1.0 \times 10^{6}$ CHO cells transfected with the normal or the mutant ALAD cDNAs. Antibody used in this study was a rabbit IgG purified from a monospecific polyclonal antibody against human $\operatorname{ALAD}(22,23)$. Detection of the specific immune complex was carried out using an enhanced chemiluminescence assay (24).

ALAD activity. ALAD activity in CHO cells was determined, as described previously (1). Human ALAD activity was specifically expressed as the activity/mass (A/M) ratio, based on the amount of human ALAD protein, as quantified by Western blot analysis.

Turnover studies. Rates of turnover of ALAD expressed in CHO cells were determined by pulse labeling. Cells were labeled with $\left[{ }^{35} \mathrm{~S}\right]-$ methionine for $16 \mathrm{~h}$. After washing, cells were incubated in the absence of the isotope for 0,3 , or $9 \mathrm{~h}$. Cells were lysed and ALAD was immuno-
A

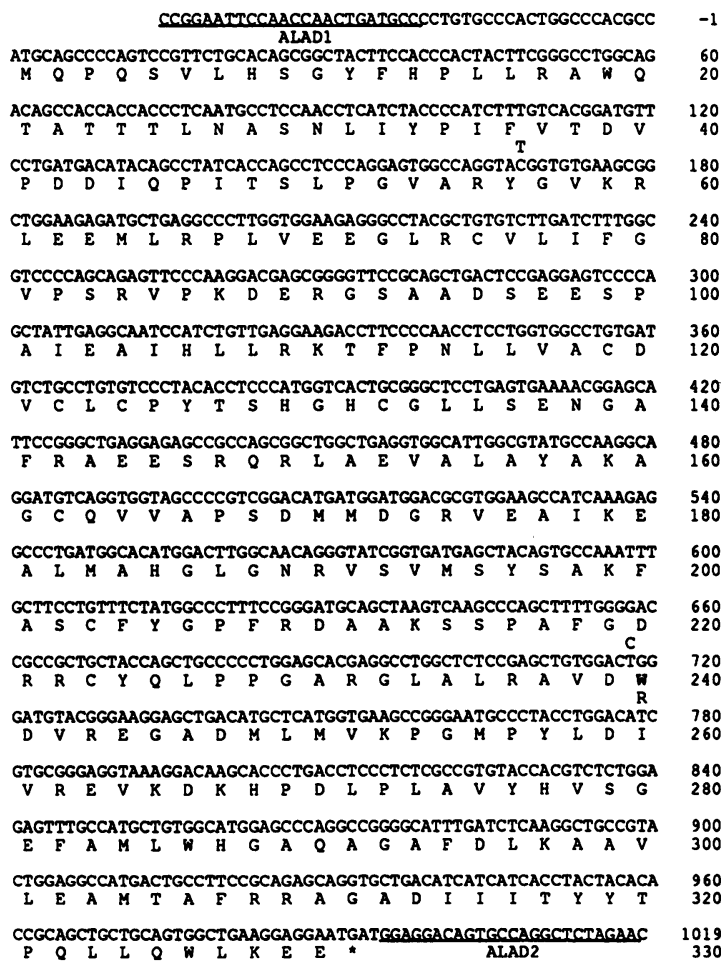

B

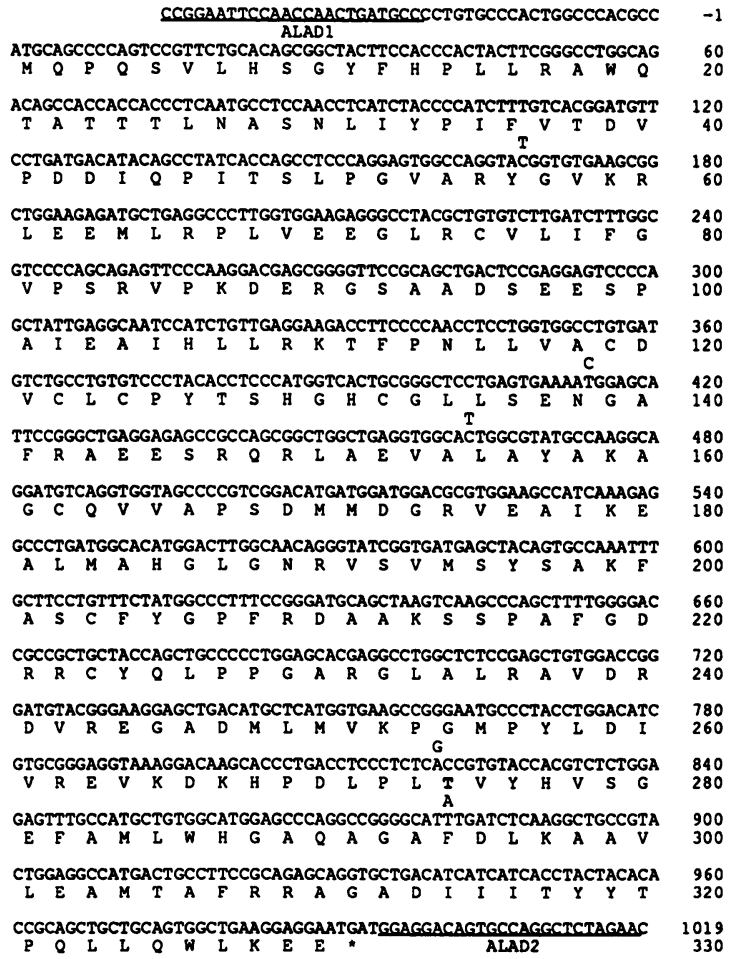

D

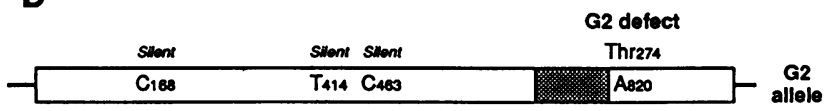

Figure 1. Nucleotide sequence of ALAD cDNAs in the proband's cells. Nucleotide residues are numbered in the $5^{\prime}$ to $3^{\prime}$ direction. Amir o acid residues deduced are expressed in one letter notation under nucleotide sequences and numbered, beginning with the initiative methionine. Normal residues for the substitution are shown above and below the nucleotide and the amino acid, respectively. The sequence of ALAD1 and that complementary to ALAD2, which were used as specific oligomers for RT-PCR, are underlined. $(A) \mathrm{G} 1 \mathrm{cDNA} ;(B) \mathrm{G} 2 \mathrm{cDNA} ;(C)$ and $(D)$ are schematic representation of the G1 and the G2 cDNA. Thick boxes and thin horizontal lines denote translated and untranslated regions, respectively. Shaded areas indicate the putative substrate-binding site of ALAD (13). 
precipitated, using the anti-human ALAD rabbit IgG, followed by Protein A-Sepharose CL-4B treatment. After washing the pellets, the radioactivity of the washed precipitates was determined, using a Tri-Carb liquid scintillation analyzer (Packard Instrument Co., Inc., Downers Grove, IL).

\section{Results}

Cloning and characterization of $c D N A$ s for the mutant ALAD alleles. The patient had ALAD activity less than $3 \%$ of normal controls, both in erythrocytes (8) and in lymphocytes (9). Immunochemical studies showed that the proband's cells contained $\approx 20 \%$ positive crossreactive immunological material (CRIM) (25), suggesting the existence of a structurally altered enzyme(s). Northern blot analysis using total RNA showed a normal size and a normal amount of ALAD mRNA (data not shown). In order to delineate the mutation of the ALAD gene in the proband, cDNAs were synthesized by MMLV reverse transcriptase from poly $(\mathrm{A})^{+}$RNA purified from EBV-transformed lymphoblastoid cells of the patient. ALAD cDNA sequences were amplified by PCR, and cloned into a pGEM4z vector. The amplification and the cloning were performed three times independently, to affirm the results. First, the G2 mutation (Fig. $1 D$ ) was identified, as described in our previous study (10). Using the allele-specific oligonucleotide for the G2 mutation (Fig. 2), three clones encoding the G2 mutation, and three G2-negative clones, were isolated from each cloning series. By nucleotide sequence analysis, the G2-negative clones were shown to encode the other mutation, termed G1 (Fig. 1 $C)$. None of the clones examined had both the $\mathrm{G} 1$ and the $\mathrm{G} 2$ mutations simultaneously, suggesting that the $\mathrm{G} 1$ and the G2 mutations were present separately on each ALAD allele. By nucleotide sequence analysis, the $\mathrm{G} 1$ clone was shown to have two point mutations, i.e., $\mathrm{T}^{168} \rightarrow \mathrm{C}$ and $\mathrm{C}^{718} \rightarrow \mathrm{T}$. The former was a silent mutation, whereas the latter accompanied an amino acid substitution $\operatorname{Arg}^{240} \rightarrow \mathrm{Trp}$; thus it is presumed to be responsible for the $\mathrm{G} 1$ defect (Fig. 1, $A$ and $C$ ). The $\mathrm{G} 2$ clone had four point mutations, but only $\mathrm{G}^{820} \rightarrow$ A produced an amino acid substitution $\mathrm{Ala}^{274} \rightarrow \mathrm{Thr}(10)$, which should thus be responsible for the $\mathrm{G} 2$ defect (Fig. 1, $B$ and $D$ ).

Phenotype studies. Using the G1-, the G2-, and the normal ALAD-specific oligomers as probes (Fig. 2), we carried out phenotype studies of the ALAD gene in the mother, a sister, and a brother of the proband. Cells from the father and the younger brother were not available for this study, for reasons beyond our control. As reported previously (10), however, RNA from the proband's cells hybridized with the oligomer specific to the G2 mutation, whereas RNA from the mother, the brother, the sister, and a normal subject did not (10). All these subjects had a normal residue at this site in the sequence (10). In contrast, all subjects from the proband's family hybridized with the oligomer specific to the G1 mutation (Fig. $3 \mathrm{~B}$ ). A normal RNA, however, did not hybridize with the $\mathrm{Gl}$ oligomer (Fig. 3 B). All these subjects had a normal residue at this site in the sequence (Figs. $3 C$ and $4 A$ ). These results indicate that the proband possesses both the $\mathrm{G} 1$ and the $\mathrm{G} 2$ mutations (Fig. $4 A$, and $B$ ), while the mother (Fig. $4 A$ ), the brother, and the sister only possess the G1 mutation. Thus the $\mathrm{G} 2$ mutation must have been transmitted by the father.

Direct sequencing of RT-PCR products of ALAD $m R N A s$. A significant frequency of misreading by Taq polymerase is known to occur during PCR (26). This problem can be circumvented, however, by directly sequencing RT-PCR products of

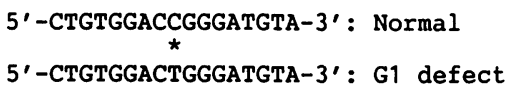

Figure 2. The ASOs for the $\mathrm{Gl}$ and the $\mathrm{G} 2$ defects. *Point mutation.

mRNAs, since any random misincorporation in an individual PCR product will not be detectable in the subsequent sequencing reaction (17). Therefore, we determined the nucleotide sequence of RT-PCR products of ALAD mRNAs to confirm the results obtained by cloning studies. Total RNAs from the proband, the mother, and a normal individual were reverse-transcribed into cDNAs. ALAD cDNAs were specifically amplified by PCR, followed by asymmetric PCR to prepare singlestranded DNAs, which were then subjected to nucleotide sequencing. Sequencing results demonstrated that the proband had sequences specific to both the $\mathrm{G} 1$ and the G2 mutations, while the mother, as expected, had only the Gl-specific sequence, and a normal individual had the normal sequence (Fig. 4, $A$ and $B$ ). These findings are consistent with the results of our ALAD phenotype studies by RT-PCR. In addition, two allelic variations associated with the $\mathrm{G} 2$ allele (Fig. $1 \mathrm{D}$ ) were confirmed by directly sequencing RT-PCR products of ALAD mRNA (Fig. 4, $C$ and $D$ ).

$A L A D$ activity expressed by the G1-ALAD and the G2$A L A D$. In order to determine the effect of the two independent single amino acid substitutions present in the $\mathrm{G} 1$ and the $\mathrm{G} 2$ alleles on ALAD activity, we performed expression studies of the normal ALAD and the mutant ALAD cDNAs by transfection in $\mathrm{CHO}$ cells. Construction of the expression vector with ALAD cDNA is shown in Fig. 5. The expressed human ALAD was quantified by Western blot analysis (24), and ALAD activity was determined by a colorimetric assay (1), and results were expressed as the A/M ratio (Fig. 6). Both the normal and the mutant ALADs were expressed as a band with $M_{\mathrm{r}} \approx 36,000$, while cells transfected with an antisense ALAD cDNA did not show any human ALAD (data not shown). CHO cells transfected with normal human ALAD cDNA expressed a high ALAD A/M ratio, while cells transfected with an antisense ALAD cDNA did not. Cells transfected with the G2 cDNA showed $\approx 0.5 \mathrm{~A} / \mathrm{M}$ ratio, while cells transfected with the $\mathrm{G} 1$ cDNA expressed only a very low $\mathrm{A} / \mathrm{M}$ ratio $(<0.1)$. Experiments were repeated at least twice for all transfectants, 1 wk apart between experiments, and similar results as above were obtained. This finding suggests that expressed phenotypes in cultured cells were essentially stable.

Rates of turnover of ALAD in CHO cells. Rates of turnover of human ALAD expressed in CHO cells were determined by pulse labeling. The G1 ALAD had a halflife similar to the normal ALAD, while the G2 ALAD had a markedly decreased halflife; $\approx 67 \%$ of the labeled G2 protein lost within $9 \mathrm{~h}$; the normal and the G1 ALAD declined at a much lesser rate $(\approx 15 \%$ at 9 h) (Fig. 7$)$.

\section{Discussion}

This report describes a complete molecular analysis of separate point mutations in each of the ALAD alleles, as well as the properties of the aberrant enzymic proteins encoded by the 

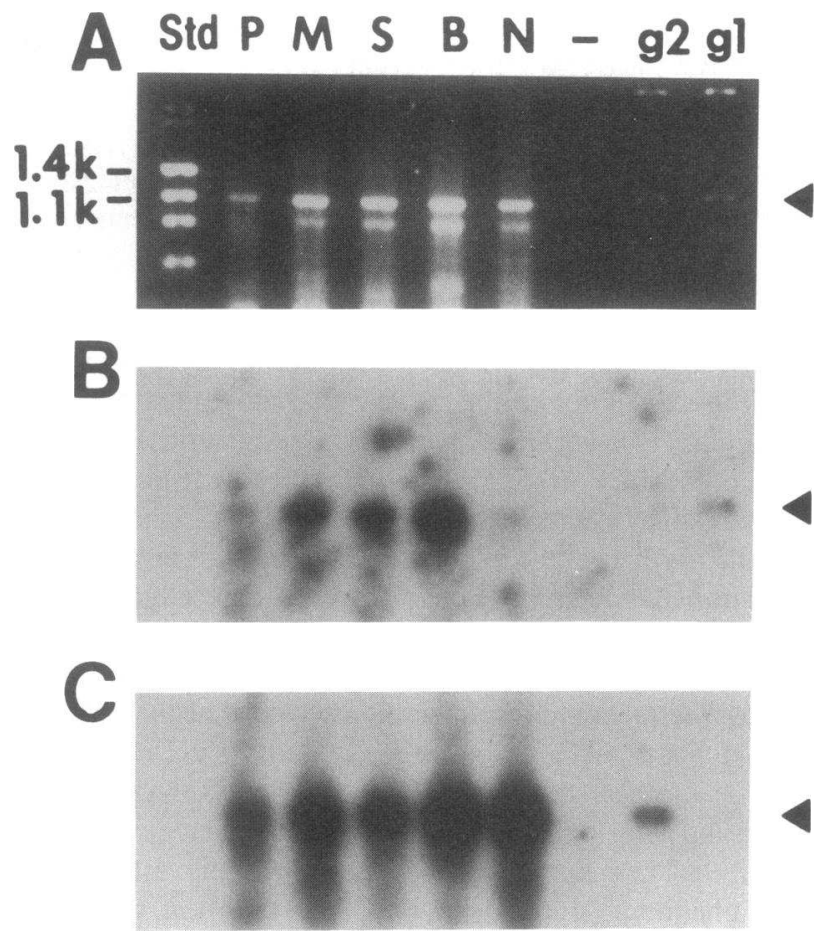

mutant ALAD genes in a patient with ADP. The occurrence of separate point mutations in the ALAD alleles was first proposed in this patient in an earlier report from our laboratory (10). There has, however, been no study to date which examined the properties of the aberrant enzymes expressed by the mutant ALAD cDNAs. This investigation has specifically examined this question by studying the properties of the enzymes expressed by transfection of mutant ALAD cDNAs into $\mathrm{CHO}$ cells. The results of our study have permitted us to unequivocally establish that the two mutant cDNAs encoded separate phenotypes of the ALAD proteins having distinctive properties.

Previous studies indicated that ALAD activity in the proband's cells was less than $3 \%$ of normal $(8,9)$, which accounted for the marked elevation of urinary ALA excretion in him (4). ALAD protein content in the proband's cells was markedly reduced, but a significant amount of CRIM was also present, suggesting that a noncatalytic enzyme was expressed (25). In the original description of this patient (8), he was considered to be homozygous for ALAD deficiency, since ALAD activity in erythrocytes was extremely low $(\approx 2 \%)$, and the parents and the siblings had enzyme activity in the range of $\approx 50 \%$ of normal (8). Our previous study (10) suggested that this patient had, in fact, two different point mutations, one in each ALAD allele. The results of the present investigation have confirmed this suggestion, and have demonstrated that the proband carries two independent single point mutations, one on each ALAD allele; $(a)$ by the use of the G1- and the G2-specific ASOs for hybridization; and $(b)$ by directly sequencing RT-PCR products of ALAD mRNAs. Thus, our data clearly indicate that this porphyric patient is characterized by compound heterozygosity for the ALAD defect. Compound heterozygosity was also reported recently in a Swedish infant with ADP, in whom point mutations different from those of our patient were described (27). However, no studies on the properties of the aberrant ALAD proteins were reported in this patient (27). Since there
Figure 3. ALAD phenotype studies in the proband's family by RT-PCR. RTPCR was carried out, as described in Methods. (A) PCR products fractionated on $1.2 \% \mathrm{wt} / \mathrm{vol}$ agarose gel. The proband's sample was less intensely amplified than others, due to an impurity in the RNA sample (unpublished data). (B) An autoradiogram of PCR products hybridized with the oligomer specific for the G1 mutation (Fig. 2). A weaker signal in the proband's sample reflected a lower yield of the RT-PCR product. Existence of the G1 mutation in the proband's sample was confirmed by directly sequencing RT-PCR products of his ALAD mRNAs (Fig. $4 A$ ). (C) An autoradiogram of PCR products hybridized with the oligomer specific for the normal sequence for the $\mathrm{G} 1$ mutation (Fig. 2). Std, size marker; $P$, proband; $M$, mother; $S$, sister, $B$, brother; $N$, normal subject; $g 2$, pADPg2.1; $g 1$, pADPg1.1 (10).

has been no consanguinity in any of the four ADP families so far studied, we suggest, on the basis of all available data, that compound heterozygosity is probably more common than is homozygosity for the ALAD defect(s) in ADP.

Phenotype studies using RT-PCR indicated that the mother, the brother, and the sister had the G1 defect (Fig. 3 B). The results with the mother, the proband, and a normal subject were also confirmed by directly sequencing RT-PCR products of ALAD mRNAs (Fig. 4). Lymphocyte ALAD activity in these subjects (28) were consistent with the heterozygosity for the $\mathrm{Gl}$ defect. Although cells from the father and the younger brother were not available for this study, the results of these studies clearly indicate that the $\mathrm{G} 2$ defect must have been transmitted to the proband from his father.

The $\mathrm{G} 1$ mutation represents $\mathrm{C}^{718} \rightarrow \mathrm{T}$, and results in the amino acid substitution, $\operatorname{Arg}^{240} \rightarrow$ Trp. This mutation occurred within the presumed substrate-binding site of the enzyme (29) (Fig. $1 C$ ), and thus would be expected to significantly influence enzyme activity. Consistent with this idea, the ALAD protein expressed by the transfection of $\mathrm{CHO}$ cells with G1 cDNA had a markedly decreased A/M ratio (<0.1) (Fig. 6). The point mutation of the $\mathrm{G} 2$ defect represents $\mathrm{G}^{820} \rightarrow \mathrm{A}$, resulting in the amino acid substitution, $\mathrm{Ala}^{274} \rightarrow$ Thr. This mutation lies outside the substrate-binding site (Fig. $1 \mathrm{D}$ ), and thus would be expected to have a lesser effect on enzyme activity than the G1 mutation. Accordingly, the A/M ratio expressed by $\mathrm{G} 2$ cDNA was only $\approx 0.5$, as compared with normal (Fig. 6). The G2 enzyme, however, had an abnormally short half life (Fig. 7), which compounded its already half decreased activity. These findings therefore account for the almost entire lack of enzyme activity in the proband's cells, and the half normal enzyme activity in cells from other family members.

ALAD purified from various mammalian sources, including humans (30), is known to be a homo-octamer, comprising 8 identical subunits of $\approx 36,000$ (1). It is interesting to specu- 
Proband

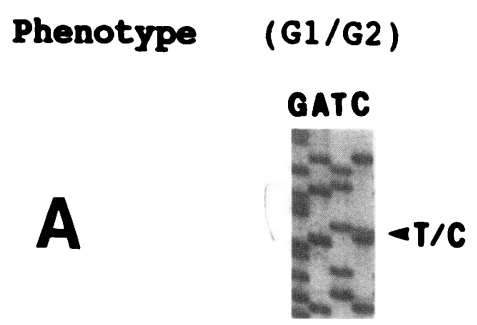

Mother

$(+/ G 1)$

GATC

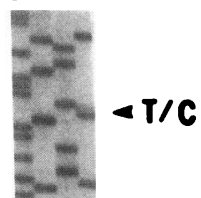

$\operatorname{Arg} 240$

Normal

: $5^{\circ}$-GAC-CGG-GAT-3'

G1 Defect: $5^{\circ}$-GAC-IGG-GAT-3'

Trp240
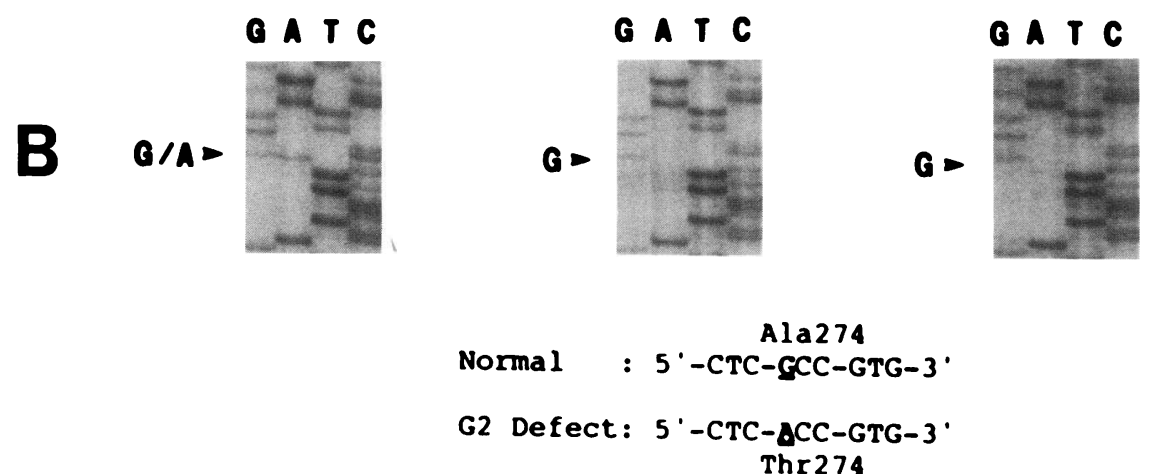

TOCA

C
T GCA

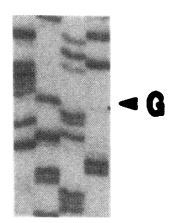

Asn

$5^{\circ}$-GAA-AAC-GGA-3'

$3^{\circ}$-CTT-TTG-CCT-5

Variation of : $5^{\prime}$-GAA-AAT-GGA-3'

G2 Allele: $3^{\circ}$-CTT-TTA-CCT-3

Asn

TOCA

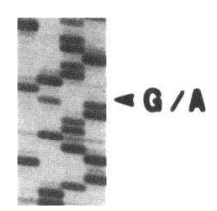

TOCA

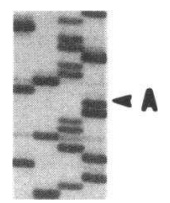

Leu

5 '-GCA-TTG-GCG-3'

$3^{\circ}-C G T-A A C-C G C-5^{\prime}$
TGCA

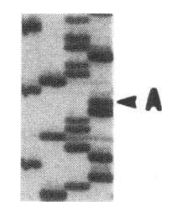

TGCA

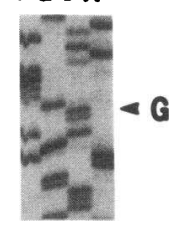

Figure 4. Direct sequencing of RT-PCR products of ALAD mRNAs from the proband, the mother, and a normal subject. Direct sequencing of RT-PCR products were carried out, as described in Methods. Sequencing ladders representing the G1 and the G2 mutations, and two variations in the $G 2$ allele are shown. Arrows indicate sites related to a defect or a variation in the sequence. (Left) Proband. (Center) Mother. (Right) Normal subject. $(A) \mathrm{G} 1$ mutation. $(B) \mathrm{G} 2$ mutation. $(C)$ A variation $\mathrm{T} 414$ of the $\mathrm{G} 2$-allele (Fig. $1 D$ ). $(D)$ A variation $\mathrm{C} 463$ of the $\mathrm{G} 2$ allele (Fig. $1 D$ ). $A$ and $B$ represent the sequence of the sense strand, while $C$ and $D$ represent the antisense strand sequence. 


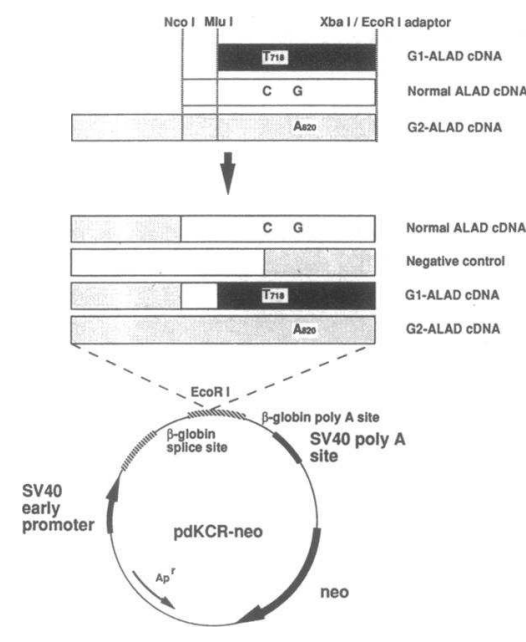

Figure 5. Construction of the expression vector with ALAD cDNAs. pdKCR-Neo is a derivative of the expression vector pKCR (18), which contained the SV40 early gene promoter, donor, and acceptor splice sites derived from the rabbit $\beta$-globin gene, as well as polyadenylation sites derived from the rabbit $\beta$-globin gene, and the SV40 early gene. A normal ALAD cDNA was reconstituted by replacing NcoI-XbaI fragment

of G2-ALAD cDNA in pADPg2.1 (10) with the normal cDNA fragment. The sequence of G2-ALAD cDNA in pADPg2.1 is shown in Fig. $1 \mathrm{~B}$. The normal ALAD cDNA was cloned from a lymphoblastoid cell line (GM8668; Human Genetic Mutant Cell Repository, Camden, PA) which had normal ALAD by RT-PCR, as described in Methods. Sequence analysis confirmed that the NcoI-Xbal fragment of the cDNA had the sequence identical to the published normal ALAD (13). G1-ALAD cDNA was reconstituted by replacing the MluI-Xbal fragment from pADPg1.1 (10). The sequence of the MluI-Xbal fragment was confirmed by sequencing. Accordingly, the reconstituted G1-ALAD cDNA had sequence shown in Fig. $1 \mathrm{~A}$. XbaI site of the 3'-end derived from ALAD2 primer was changed into EcoRI with XbaI-XmnI and EcoRI-XmnI adapters (Clonetech, Palo Alto, $C A$ ). These cDNAs were introduced into the EcoRI site of the 3 rd exon of rabbit $\beta$-globin. A normal ALAD cDNA recombinant, introduced in opposite direction into pdKCR-Neo, was used as a negative control.

late how many ALAD subunits exist in the proband's holoenzyme that are derived from each ALAD allele; however, this question cannot be dealt with at present, since there is no structural information on the assembly of heteroallelic ALAD sub-

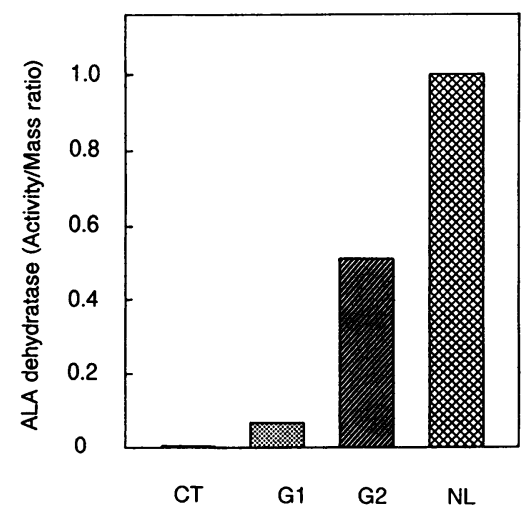

Figure 6. Human ALAD expression in CHO cells. Transfection of $\mathrm{CHO}$ cells with ALAD cDNAs, quantitation of the human ALAD protein expressed in cells, and assays on ALAD activity were carried out, as described in Methods. The basal level of ALAD activity in $\mathrm{CHO}$ cells was negligible, and the amount of CHO ALAD

protein quantitated by Western blot analysis using a rabbit IgG vs. mouse ALAD (23) was comparable for all samples (data not shown). Data are the mean of triplicate assays. Human ALAD expressed by transfection with anti-sense ALAD cDNA $(C T), \mathrm{G} 1 \mathrm{cDNA}(G 1), \mathrm{G} 2$ cDNA (G2), ALAD cDNA ( $N L)$, and ALAD purified from normal human erythrocytes $(A D)$.

An A/M ratio of $1.0(N L)$ corresponds to $5.2 \mathrm{nmol}$ porphobilinogen formed/microgram protein, $h, 37^{\circ} \mathrm{C}$.

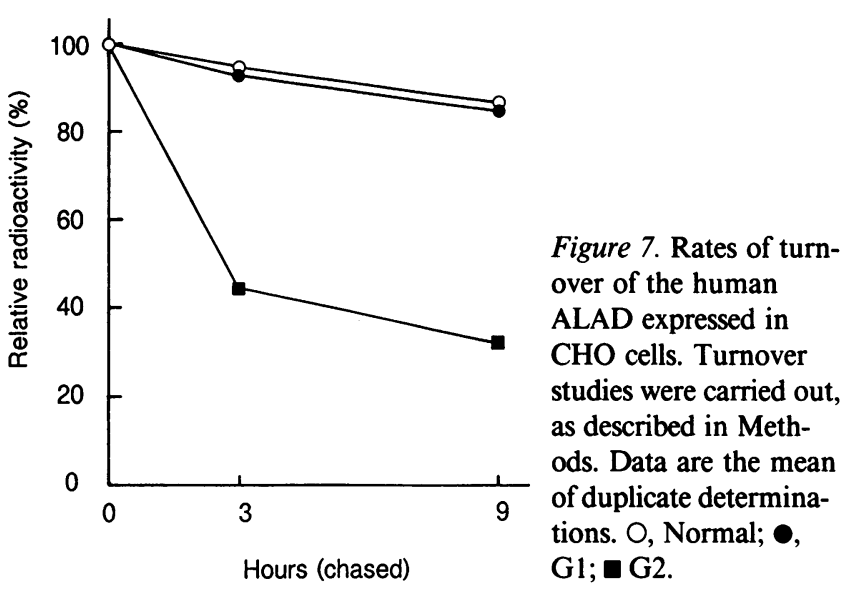

units. The availability of $\mathrm{CHO}$ cells expressing a mutant homooctameric G1 or a G2 enzyme, as shown in this study, should prove useful in resolving this and related aspects of ALAD defects.

The proband suffered severe porphyric symptoms presumably because of his marked ALAD deficiency; other members of the family displayed no symptomatology, probably because a half-normal ALAD activity is entirely sufficient for normal heme synthesis $(4,7)$. The $\approx 20 \%$ CRIM in the proband $(25)$ is likely to reflect the protein product of the $\mathrm{G} 1$ allele, rather than that of the $\mathrm{G} 2$ allele, since the $\mathrm{G} 2$ product is more unstable than the G1 product. The fact that the patient survived, however, may be due to the residual activity contributed by the G2 enzyme, since the $G 1$ enzyme was essentially nonfunctional (Fig. 6). A more marked enzyme deficiency may not be compatible with life, since a couple known to be heterozygous for ALAD deficiency $(\approx 50 \%)$ had experienced four successive spontaneous abortions (31).

Since all individuals so far identified with $\approx 50 \%$ ALAD activity were all clinically unaffected $(2,8,27,31,32)$, these defects must be widely found as germline mutations. Including the two point mutations described in this study, at least four distinct germline mutations of ALAD have been recognized to date $(10,27)$. A Swedish study raised the possibility that the frequency of heterozygous carriers of ALAD deficiency could be as high as $2 \%$ in a random normal population (31). Although heterozygotes with such mutations do not normally display clinical consequences, they may represent a population which could be at a high risk for the toxic effects of chemicals, e.g., iron (33), lead (5), trichlorethylene (34), and styrene (35), which are known to adversely affect ALAD activity (36). For example, it is known that individuals with heterozygous ALAD deficiency and with high normal blood lead levels display evidence of subclinical lead poisoning manifest by excessive porphyrin elevation in blood (37). The implications of heterozygosity for ALAD deficiency may thus bear significantly on the susceptibility of affected individuals to toxic effects of environmental chemicals that interact with this enzyme.

\section{Acknowledgments}

We are grateful to Drs. Shinzo Oikawa and Hiroshi Nakazato, Suntory Institute for Biomedical Research, and Dr. Franca LaBella, Rockefeller University, for providing us with pdKCR-Neo and CHO cells, respec- 
tively. The excellent technical assistance of Ms. Luba Garbachewski is gratefully acknowledged.

This study was supported in part by grants from U.S.P.H.S. (DK32890), Suntory Fund for Biomedical Research, Deutsche Forschungsgemeinschaft (DO 134), and gifts from the Ogden Corporation and the Renfield Foundation.

\section{References}

1. Sassa, S. 1982. Delta-aminolevulinic acid dehydratase assay. Enzyme (Basel). 28:133-145.

2. Bird, T. D., P. Hamernyik, J. Y. Nutter, and R. F. Labbe. 1979. Inherited deficiency of delta-aminolevulinic acid dehydratase. Am. J. Hum. Genet. 31:662668.

3. Bonkovsky, H. L. 1982. Porphyrin and heme metabolism and the porphyrias. In Hepatology: A Textbook of Liver Disease. D. Zakim, and T. D. Boyer, editors. Saunders, Philadelphia. 351-393.

4. Kappas, A., S. Sassa, R. A. Galbraith, and Y. Nordmann. 1989. The porphyrias. In The Metabolic Basis of Inherited Disease. C. R. Scriver, A. L. Beaudet, W. S. Sly, and D. Valle, editors. McGraw-Hill Book Co., New York. 1305-1365.

5. Sassa, S., S. Granick, and A. Kappas. 1975. Environmental chemical and genetic effects on heme biosynthesis in the human red cell. Ann. NY Acad. Sci. 244:419-440.

6. Lindblad, B., S. Lindstedt, and G. Steen. 1977. On the genetic defects in hereditary tyrosinemia. Proc. Natl. Acad. Sci. USA. 74:4641-4645.

7. Sassa, S., and A. Kappas. 1983. Hereditary tyrosinemia and the heme biosynthetic pathway. Profound inhibition of delta-aminolevulinic acid dehydratase activity by succinylacetone. J. Clin. Invest. 71:625-634.

8. Doss, M., H. G. Benkmann, and H. W. Goedde. 1986. delta-Aminolevulinic acid dehydrase (porphobilinogen synthase) in two families with inherited enzyme deficiency. Clin. Genet. 30:191-198.

9. Sassa, S., H. Fujita, M. Doss, A. Hassoun, L. Verstraeten, R. Mercelis, and A. Kappas. 1991. Hereditary hepatic porphyria due to homozygous $\delta$-aminolevulinic acid dehydratase deficiency: studies in lymphocytes and erythrocytes. Eur. $J$. Clin. Invest. 21:244-248.

10. Ishida, N., H. Fujita, T. Noguchi, M. Doss, A. Kappas, and S. Sassa. 1990. Message amplification phenotyping of an inherited $\delta$-aminolevulinate dehydratase deficiency in a family with acute hepatic porphyria. Biochem. Biophys. Res. Commun. 172:237-242.

11. Chirgwin, J. M., A. E. Przybyla, R. J. MacDonald, and W. J. Rutter. 1979. Isolation of biologically active ribonucleic acid from sources enriched in ribonuclease. Biochemistry. 18:5294-5299.

12. Aviv, H., and P. Leder. 1972. Purification of biologically active globin messenger RNA by chromatography on oligothymidylic acid-cellulose. Proc. Natl. Acad. Sci. USA. 69:1408-1412.

13. Wetmur, J. B., D. F. Bishop, C. Cantelmo, and R. J. Desnick. 1986. Human delta-aminolevulinate dehydratase: nucleotide sequence of a full length cDNA clone. Proc. Natl. Acad. Sci. USA. 83:7703-7707.

14. Sanger, F., S. Nicklen, and A. R. Coulson. 1977. DNA sequencing with chain-terminating inhibitors. Proc. Natl. Acad. Sci. USA. 74:5463-5467.

15. Messing, J., R. Crea, and P. H. Seeburg. 1981. A system for shotgun DNA sequencing. Nucleic Acids Res. 9:309-321.

16. Tabor, S., and C. C. Richardson. 1990. DNA sequence analysis with a modified bacteriophage T7 DNA polymerase. Effect of pyrophosphorolysis and metal ions. J. Biol. Chem. 265:8322-8328.

17. Gyllensten, U. B., and H. A. Erlich. 1988. Generation of single-stranded DNA by the polymerase chain reaction and its application to direct sequencing of the HLA-DQA locus. Proc. Natl. Acad. Sci. USA. 85:7652-7656.

18. O'Hare, K., C. Benoist, and R. Breathnach. 1981. Transformation of mouse fibroblasts to methotrexate resistance by a recombinant plasmid express- ing a prokaryotic dihydrofolate reductase. Proc. Natl. Acad. Sci. USA 78:15271531.

19. Oikawa, S., C. Inuzuka, M. Kuroki, Y. Matsuoka, G. Kosaki, and H. Nakazato. 1989. Cell adhesion activity of non-specific cross-reacting antigen (NCA) and carcinoembryonic antigen (CEA) expressed on CHO cell surface: homophilic and heterophilic adhesion. Biochem. Biophys. Res. Commun. 164:39-45.

20. Nikaido, T., A. Shimizu, N. Ishida, H. Sabe, K. Teshigawara, M. Maeda, T. Uchiyama, J. Yodoi, and T. Honjo. 1984. Molecular cloning of cDNA encoding human interleukin-2 receptor. Nature (Lond.). 311:631-635.

21. Tsujimoto, M., H. Adachi, S. Kodama, N. Tsuruoka, Y. Yamada, S. Tanaka, S. Mita, and K. Takatsu. 1989. Purification and characterization of recombinant human interleukin 5 expressed in Chinese hamster ovary cells. $J$. Biochem. (Tokyo). 106:23-28.

22. Chang, C. S., and S. Sassa. 1985. delta-Aminolevulinate dehydratase in human erythroleukemia cells: an immunologically distinct enzyme. Blood. 65:939-944.

23. Chang, C. S., S. Sassa, and D. Doyle. 1984. An immunological study of delta-aminolevulinic acid dehydratase: specificity consistent with the phylogeny of species. Biochim. Biophys. Acta. 797:297-301.

24. Technical Manual. 1990. ECL Western blotting detection system. Amersham International plc, Amersham, UK. 1-33.

25. de Verneuil, H., M. Doss, N. Brusco, C. Beaumont, and Y. Nordmann. 1985. Hereditary hepatic porphyria with delta-aminolevulinate dehydratase deficiency: immunologic characterization of the non-catalytic enzyme. Hum. Genet. 69:174-177.

26. Tindall, K. R., and T. A. Kunkel. 1988. Fidelity of DNA synthesis by the Thermus aquaticus DNA polymerase. Biochemistry. 27:6008-6013.

27. Plewinska, M., S. Thunell, L. Holmberg, J. G. Wetmur, and R. J. Desnick. 1991. $\delta$-Aminolevulinate dehydratase deficient porphyria: identification of the molecular lesions in a severely affected homozygotes. Am. J. Hum. Genet. 49:167-174.

28. Wetmur, J. G., D. F. Bishop, L. Ostasiewicz, and R. J. Desnick. 1986. Molecular cloning of a cDNA for human delta-aminolevulinate dehydratase. Gene (Amst.). 43:123-130.

29. Anderson, P. M., and R. J. Desnick. 1979. Purification and properties of delta-aminolevulinate dehydratase from human erythrocytes. J. Biol. Chem. 254:6924-6930.

30. Thunell, S., L. Holmberg, and J. Lundgreen. 1987. Aminolevulinate dehydratase porphyria in infancy. A clinical and biochemical study. J. Clin. Chem. Clin. Biochem. 25:5-14.

31. Fujita, H., S. Sassa, J. Lundgren, L. Holmberg, S. Thunell, and A. Kappas. 1987. Enzymatic defect in a child with hereditary hepatic porphyria due to homozygous delta-aminolevulinic acid dehydratase deficiency: immunochemical studies. Pediatrics. 80:880-885.

32. Bonkovsky, H. L., J. F. Healey, B. Lincoln, B. R. Bacon, D. F. Bishop, and G. H. Elder. 1987. Hepatic heme synthesis in a new model of experimental hemochromatosis: studies in rats fed finely divided elemental iron. Hepatology. 7:1195-1203

33. Fujita, H., A. Koizumi, M. Yamamoto, M., Kumai, T. Sadamoto, and M Ikeda. 1984. Inhibition of delta-aminolevulinate dehydratase in trichloroethylene-exposed rats, and the effects on heme regulation. Biochim. Biophys. Acta. 800:1-10.

34. Fujita, H., A. Koizumi, N. Hayashi, and M. Ikeda. 1986. Reduced synthesis of 5-aminolevulinate dehydratase in styrene-treated rats. Biochim. Biophys. Acta. 867:89-96.

35. Sassa, S., H. Fujita, and A. Kappas. 1989. Genetic and chemical influences on heme biosynthesis. In Highlights of Modern Biochemistry. A. Kotyk, J. Skoda, V. Paces, and V. Kostka, editors. VSP Int. Science Publishers, Zeist, Netherlands. 329-337.

36. Doss, M., F. Laubenthal, and M. Stoeppler. 1984. Lead poisoning in inherited delta-aminolevulinic acid dehydratase deficiency. Int. Arch. Occup. Environ. Health. 54:55-63. 